\title{
Model for twin electromagnons and magnetically induced oscillatory polarization in multiferroic $\mathrm{RMnO}_{3}$
}

\author{
Markku P. V. Stenberg® and Rogério de Sousd \\ Department of Physics and Astronomy, University of Victoria, Victoria, British Columbia, Canada V8W $3 P 6$
}

(Dated: November 27, 2018)

\begin{abstract}
We propose a model for the pair of electromagnon excitations observed in the class of multiferroic materials $R \mathrm{MnO}_{3}$ ( $R$ is a rare-earth ion). The model is based on a harmonic cycloid ground state interacting with a zone-edge magnon and its twin excitation separated in momentum space by two times the cycloid wave vector. The pair of electromagnons is activated by cross coupling between magnetostriction and spin-orbit interactions. Remarkably, the spectral weight of the twin electromagnon is directly related to the presence of a magnetically induced oscillatory polarization in the ground state. This leads to the surprising prediction that $\mathrm{TbMnO}_{3}$ has an oscillatory polarization with amplitude 50 times larger than its uniform polarization.
\end{abstract}

PACS numbers: 75.80.+q, 78.20.Ls, 71.70.Ej, 75.30.Et

\section{INTRODUCTION}

The coexistence of magnetic and ferroelectric phases in multiferroic materials gives rise to important effects related to cross correlation between order parameters and external fields $: \frac{1,2}{}$ A notable consequence is that the elementary excitations are not purely magnetic or ferroelectric in character. The spin waves are admixed with vibrational modes of the electric polarization, giving rise to electric dipole active magnons, the so-called electromagnons. Electromagnons were postulated to exist in 1969 by Bary'akhtar and Chupis ${ }^{\underline{3}}$ but their existence was confirmed only recently by sensitive optical experiments in the far infrared $4,5,6,7,8,9$ combined with neutron scattering 10

Apart from its fundamental importance, the electromagnon spectra gives invaluable information on how magnetism couples to ferroelectricity. The class of perovskite manganites $R \mathrm{MnO}_{3}$ (RMO) is playing a major role in this respect. Here $R$ is a rare-earth ion such as $\mathrm{Gd}, \mathrm{Tb}, \mathrm{Dy}$, or mixtures between them (e.g., $\mathrm{Gd}_{x} \mathrm{~Tb}_{1-x}$, etc.). At low temperatures $(T \sim 20 \mathrm{~K})$ the $\mathrm{Mn}$ spins are ordered in a spiral state with period incommensurate with the lattice $\underline{\underline{11}}$ The spin spiral is of the cycloid type. This breaks space inversion and gives rise to a ferroelectric moment along one of the directions in the cycloid plane $\frac{12}{2}$ At the microscopic level, this ferroelectric moment originates from Dzyaloshinskii-Moriya (DM) coupling, $12,13,14,15$

Nevertheless, contrary to predictions based on DM coupling,,$\frac{16}{=}$ electromagnons in RMO are observed only when the electric field of light is along the crystallographic direction $\hat{a} \cdot \frac{7.8,9,17}{1}$ Moreover, for all ions $R$, two electromagnons are always observed, $\stackrel{18}{\underline{2}}$ Recently, the origin of one of these electromagnons was explained by Aguilar et al.,,$\frac{7.8}{3}$ who pointed out that the high frequency electromagnon was a zone-edge magnon activated by pure magnetostriction. However, the origin of the lowfrequency electromagnon remains unknown.

A recent theory 19 shows that the anharmonicity of a distorted cycloid ground state gives rise to multiple elec- tromagnons that were observed using Raman scattering in $\mathrm{BiFeO}_{3} \stackrel{6}{*}$ However, the anharmonicity mechanism cannot explain the pair of electromagnons in RMO because the degree of anharmonicity detected by neutron diffraction is too low in these materials $\underline{11}$

Here we propose a model that is able to explain the double electromagnon feature observed in RMO. We show that a simple harmonic cycloid ground state with wave vector $Q$ has a special pair of twin electromagnon excitations, located at the zone edge and $2 Q$ away from it. Remarkably, the activation of the latter electromagnon is directly related to the presence of an oscillatory polarization with wave vector $2 Q$ in the ground state.

\section{MODEL}

The usual phenomenological Landau theory is based on a free-energy expansion into powers of the spatial derivatives of the order parameters $\stackrel{20}{=}$ Therefore, the Landau approach $3,12,19,21$ cannot describe large wave vector excitations such as zone-edge magnons. Here we adopt a model that is more microscopic than the Landau theory. Our model Hamiltonian consists of three distinct contributions, $H=H_{S}+H_{\mathrm{ph}}+H_{\mathrm{me}}$. The first contribution describes spin frustration in RMO, $, 7,8,10,11,22$

$$
H_{S}=\sum_{n, m} J_{n, m} \hat{\mathbf{S}}_{n} \cdot \hat{\mathbf{S}}_{m}+D \sum_{n}\left(\hat{\mathbf{S}}_{n} \cdot \hat{a}\right)^{2} .
$$

Here $J_{n, m}$ is the exchange coupling between Mn spins at positions $\boldsymbol{R}_{n}$ and $\boldsymbol{R}_{m}$, and $D$ is a single-ion anisotropy along the crystallographic direction $\hat{a}$ (we assume $D>0$, i.e., easy-plane anisotropy). RMO has an orthorhombic lattice, with an orthogonal system of crystal axis $\hat{a}, \hat{b}, \hat{c}$ and unequal bond lengths $a \neq b \neq c$. There are four Mn spins per unit cell; spins 1 and 2 lie in the $a b$ planes, with spins 3 and 4 a distance $c / 2$ above them. The coupling between nearest neighbor spins in the $a b$ planes is ferromagnetic, and will be denoted by $J_{0}$; the coupling between $a b$ layers along the $\hat{c}$ direction is antiferromagnetic and is denoted by $J_{c}$. Spin frustration arises due 


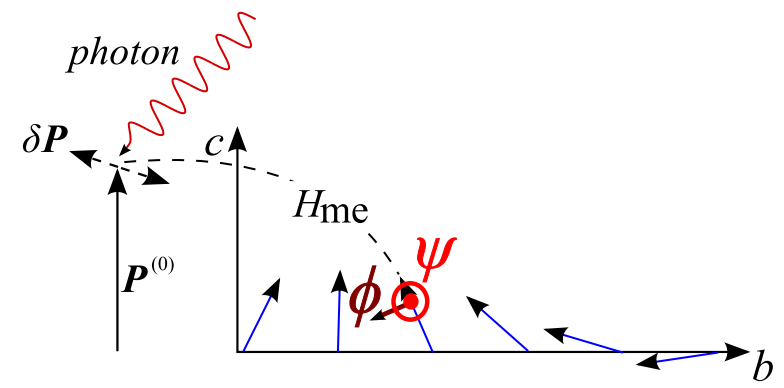

FIG. 1: (Color online) Coupled spin waves and optical phonons in the cycloid ground state. In-plane spin fluctuations are denoted by $\phi$, while out-of-plane spin fluctuations are denoted by $\psi$.

to coupling between next-nearest neighbors along the $\hat{b}$ direction; this is antiferromagnetic and denoted by $J_{2 b}$. When the stability condition $J_{2 b}>-J_{0} / 2$ is satisfied, the ground state is a $b c$ cycloid $13,14,15$ as observed in experiments, $, 1,11$

$$
\boldsymbol{S}_{0}(\mathbf{R})= \pm S[\cos (\mathbf{Q} \cdot \mathbf{R}) \hat{b}+\sin (\mathbf{Q} \cdot \mathbf{R}) \hat{c}] .
$$

Here $\boldsymbol{Q}=Q \hat{b}$ is the cycloid wave vector, with $\cos (Q b / 2)=-J_{0} /\left(2 J_{2 b}\right)$. The upper $(+)$ sign applies to $a b$ layer spins 1 and 2 , whereas the lower $(-)$ sign applies to spins 3 and 4 in the neighboring $a b$ layers immediately above and below them. Figure 1 depicts the cycloid ground state. The second ingredient of our model is the lattice fluctuation Hamiltonian,

$$
H_{\mathrm{ph}}=\frac{1}{2} m^{*} \sum_{n}\left(\dot{\boldsymbol{x}}_{n}^{2}+\omega_{0}^{2} \boldsymbol{x}_{n}^{2}\right)-e^{*} \sum_{n} \boldsymbol{x}_{n} \cdot \boldsymbol{E},
$$

where $\boldsymbol{x}_{n}$ is a relative displacement between cations and anions within each unit cell. Here $e^{*}$ is the Born effective charge, $m^{*}$ is an effective mass, and $\omega_{0}$ is the (bare) phonon frequency. The modes $\boldsymbol{x}_{n}$ are directly related to the polarization order parameter in Fourier space, $\boldsymbol{P}_{\boldsymbol{q}}(\omega)=\frac{e^{*}}{N v_{0}} \int \mathrm{d} t \sum_{n} \mathrm{e}^{i\left(\boldsymbol{q} \cdot \boldsymbol{R}_{n}-\omega t\right)} \boldsymbol{x}_{n}$, where $v_{0}$ is the volume of the unit cell. The electric field of light, $\boldsymbol{E} \mathrm{e}^{-i \omega t}$, couples linearly to the $q=0$ polarization, $\boldsymbol{P}_{0}(\omega)=\chi_{0}(\omega) \boldsymbol{E}$, with electric susceptibility $\chi_{0}(\omega)=$ $\left(e^{* 2}\right) /\left[\left(m^{*} v_{0}\right)\left(\omega_{0}^{2}-\omega^{2}\right)\right]$.

The third contribution $H_{\text {me }}$ models the coupling between the spin degrees of freedom and the lattice. Following the experimental observation that electromagnons are only excited when the electric field is along $\hat{a}, \underline{7,8,9,17}$ we consider the interaction,

$$
\begin{aligned}
H_{\mathrm{me}}= & e^{*} \sum_{n} x_{n}^{a}\left[g_{c}\left(\hat{S}_{1, n}^{c}-\hat{S}_{1, n+b}^{c}\right)\left(\hat{S}_{2, n}^{c}+\hat{S}_{2, n+a}^{c}\right)\right. \\
& +g_{b}\left(\hat{S}_{1, n}^{b}-\hat{S}_{1, n+b}^{b}\right)\left(\hat{S}_{2, n}^{b}+\hat{S}_{2, n+a}^{b}\right) \\
& +(1 \rightarrow 3,2 \rightarrow 4)],
\end{aligned}
$$

which is invariant under the Pbnm space-group operations of RMO. Here $g_{c} \neq g_{b}$ are coupling constants with dimension of electric fields; we neglect the components proportional to $g_{a}$ because these play no role in the discussion below. Remarkably, Eq. (4) is not rotationally invariant in spin operators. Therefore it differs in an important way from the pure magnetostrictive coupling considered previously $\underline{\underline{7}}$ The absence of rotational invariance in magnetoelectric coupling is a direct consequence of cross coupling between magnetostriction and spin-orbit interactions, and should be common property of all multiferroics with orthorhombic lattice (this follows from symmetry since $a \neq b \neq c$ precludes rotations that take one axis into the other). Later we will explain why the experimental observations favor this coupling over other symmetry-allowed explanations.

It turns out that Eq. (4) gives rise to a remarkable static effect. Minimizing $H_{\mathrm{ph}}$ with respect to $\boldsymbol{x}_{n}$, and plugging in the cycloidal spin order we get

$$
\frac{e^{*} \boldsymbol{x}_{n}}{v_{0}}=P_{\mathrm{IOP}} \sin [(2 n+1) Q b] \hat{a} .
$$

Hence the local polarization per unit cell is oscillatory with wave vector $2 Q$; the amplitude for the incommensurate oscillatory polarization (IOP) is given by

$$
P_{\mathrm{IOP}}=4\left(g_{b}-g_{c}\right) \chi_{0} S^{2} \sin \left(\frac{Q b}{2}\right) .
$$

Remarkably, the presence of the IOP is directly related to the lack of rotational invariance in Eq. (4), i.e., the fact that $g_{b} \neq g_{c}$.

It is well known that $\mathrm{TbMnO}_{3}$ has a magneticallyinduced lattice modulation with wave vector $2 Q$ in the cycloidal phase $\stackrel{1}{\underline{n}}$ However, we are not aware of any claims that such a modulation leads to oscillatory electric polarization. The main result of this paper is to show that this oscillatory polarization is directly related to the spectral weight of the twin electromagnon; hence it can be detected by optical experiments.

\section{TWIN ELECTROMAGNON EXCITATIONS}

We now consider the elementary excitations of the coupled spin-phonon system. The spin excitations $\delta \hat{\boldsymbol{S}}=$ $\hat{\boldsymbol{S}}-\boldsymbol{S}_{0}$ are parametrized as follows,

$$
\delta \hat{\boldsymbol{S}}_{i, n}=\hat{\psi}_{i, n} \hat{a} \pm \hat{\phi}_{i, n}\left[\cos \left(\boldsymbol{Q} \cdot \boldsymbol{R}_{i, n}\right) \hat{c}-\sin \left(\boldsymbol{Q} \cdot \boldsymbol{R}_{i, n}\right) \hat{b}\right]
$$

where the sign convention is the same as in Eq. (2). Here the operators $\hat{\psi}$ describe spin fluctuations out of the cycloidal plane, and $\hat{\phi}$ denotes in-plane (tangential) fluctuations (Fig. 11). We carry out a mean-field expansion of the Hamiltonian $H$ about its equilibrium value by keeping only terms quadratic in the fluctuation operators, e.g., $\delta P^{2}, \hat{\phi}^{2}, \delta P \hat{\psi}$, etc. Using the canonical commutation relations $\left[\hat{\phi}_{j, n}, \hat{\psi}_{k, m}\right]=i \delta_{j k} \delta_{n m} S$, we derive the 


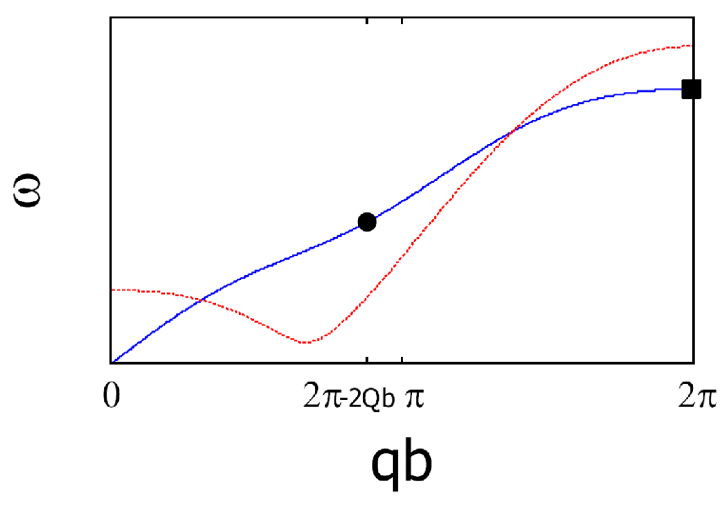

FIG. 2: (Color online) Typical dispersion curves along the cycloid direction $\hat{b}$ for the cyclon (blue solid) and extra-cyclon (red dashed) modes in a $b c$ cycloid. Our theory gives rise to twin electromagnons depicted by a circle and a square. When the magnetoelectric interaction is rotationally invariant $\left[g_{b}=\right.$ $g_{c}$ in Eq. (4)], only the one denoted by a square is excited; in the absence of rotational invariance, both electromagnons are excited. Here $Q$ is the magnitude of the cyclon wave vector and $b$ the lattice constant along $\hat{b}$ axis.

coupled equations of motion for spin and polarization,

$$
\begin{gathered}
\left(\omega^{2}-\Omega_{\mathrm{C}, \mathrm{q}}^{2}\right)\left(\phi_{1 q}+\phi_{2 q}+\phi_{3 q}+\phi_{4 q}\right)=\Gamma_{q-k_{0}}, \\
\left(\omega^{2}-\Omega_{\mathrm{C}, \mathrm{q}+\mathrm{k}_{0}}^{2}\right)\left(\phi_{1 q}-\phi_{2 q}+\phi_{3 q}-\phi_{4 q}\right)=\Gamma_{q}, \\
\left(\omega^{2}-\Omega_{\mathrm{EC}, \mathrm{q}}^{2}\right)\left(\phi_{1 q}+\phi_{2 q}-\phi_{3 q}-\phi_{4 q}\right)=0 \\
\left(\omega^{2}-\Omega_{\mathrm{EC}, \mathrm{q}+\mathrm{k}_{0}}^{2}\right)\left(\phi_{1 q}-\phi_{2 q}-\phi_{3 q}+\phi_{4 q}\right)=0 .
\end{gathered}
$$

Here $\phi_{i q}$ are Fourier transforms of the fields $\phi_{i n}$; identical equations hold for the fields $\psi_{i n} \stackrel{23}{=}$ There are four spin-wave modes, each transforming according to four different $1 d$ representations of the Pbnm space-group. The second mode [Eq. $8 \mathrm{~b}$ )] is related to the first mode by $\boldsymbol{q} \rightarrow \boldsymbol{q}+\boldsymbol{k}_{0}$, with $\boldsymbol{k}_{0}=(2 \pi / b) \hat{b}$ the Brillouin zone edge (the lattice has periodicity $b / 2$ along $\hat{b}$ ). This corresponds to the fact that "anti-phase" fluctuations of the neighboring spins with the wave vector $q$ are equivalent to "in-phase" fluctuations at $q+2 \pi / b$. Their dispersion satisfies $\Omega_{\mathrm{C}, \mathrm{q} \rightarrow 0} \rightarrow 0$, reflecting the phase sliding symmetry of the cycloid. Adding a constant phase $\phi$ to Eq. (2) yields a different ground state with the same energy. At $q=0$ the first mode is a pure phase fluctuation $\left(\phi_{i n}\right.$ is the same for all $i$ and $\psi_{i n}=0$ ). For this reason this mode will be referred as a cyclon, with dispersion $\Omega_{\mathrm{C}, \mathrm{q}}$. The second mode is naturally called a zone-edge cyclon. Similarly, the fourth mode can be obtained from the third by the same translation in $q$ space, $\boldsymbol{q} \rightarrow \boldsymbol{q}+\boldsymbol{k}_{0}$. However, the third and fourth modes are gapped (gap proportional to $J_{c}$ ). These modes will be denoted extra cyclons. The (bare) magnon dispersions are shown in Fig. 2, For $\boldsymbol{q}$ a)

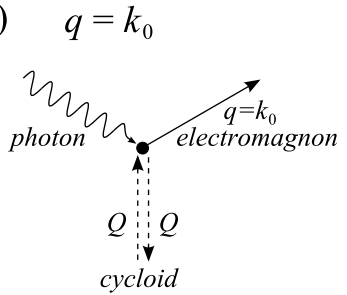

b)

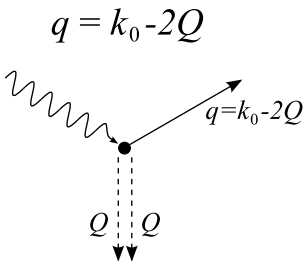

FIG. 3: Diagrams for twin electromagnon excitation by light, involving momentum exchange with the cycloidal ground state. (a) Zone-edge electromagnon at $q=k_{0}$ and (b) its twin at $q=k_{0}-2 Q$. The fact that momentum is not conserved by $k_{0}=2 \pi / b$ reflects the underlying lattice symmetry, which produces spin-wave modes connected by $q \rightarrow q+k_{0}$.

along $\hat{b}$ they are given by

$$
\begin{aligned}
\Omega_{\mathrm{C}, \mathrm{q}}^{2}= & \frac{4 S^{2}}{\hbar^{2}}\left[\left(J_{0}+2 J_{2 b} \cos \frac{q b}{2}\right)^{2}+2 J_{2 b}\left(D+2 J_{c}\right)\right] \\
& \times\left(4 \cos ^{2} \frac{q b}{4}-\frac{J_{0}^{2}}{J_{2 b}^{2}} \cos \frac{q b}{2}\right) \sin ^{2} \frac{q b}{4}, \\
\Omega_{\mathrm{EC}, \mathrm{q}}^{2}= & \frac{4 S^{2}}{\hbar^{2}}\left[\left(J_{0}+2 J_{2 b} \cos \frac{q b}{2}\right)^{2}+2 J_{2 b} D\right] \\
\times & {\left[\left(4 \cos ^{2} \frac{q b}{4}-\frac{J_{0}^{2}}{J_{2 b}^{2}} \cos \frac{q b}{2}\right) \sin ^{2} \frac{q b}{4}+\frac{J_{c}}{J_{2 b}}\right](9 \mathrm{~b}) }
\end{aligned}
$$

Typical values for the exchange couplings are $J_{0} \sim-0.4$ $\mathrm{meV}, J_{2 b} \sim 0.3-0.4 \mathrm{meV}$, and $J_{c} \sim 0.5-1 \mathrm{meV} \stackrel{\underline{8}}{\mathrm{In}} \mathrm{In}$ terestingly, only the zone-edge cyclon is coupled to the polarization. This occurs through the dynamical magnetoelectric field,

$$
\begin{aligned}
\Gamma_{q}= & \frac{8 v_{0}}{\hbar^{2}} S^{2} \sin \left(\frac{Q b}{2}\right)\left\{2 J_{0}\left[\cos \left(\frac{q b}{2}\right)+\cos \left(\frac{Q b}{2}\right)\right]\right. \\
& \left.-J_{2 b}[\cos (q b)-\cos (Q b)]-\left(D+2 J_{c}\right)\right\} \\
& \times\left\{2\left(g_{b}+g_{c}\right) P_{q}^{a}(\omega)\right. \\
& \left.-\left(g_{b}-g_{c}\right)\left[P_{q-2 Q}^{a}(\omega)+P_{q+2 Q}^{a}(\omega)\right]\right\} .
\end{aligned}
$$

Similarly, the equation of motion for the polarization only couples $P_{0}^{a}$ to the zone-edge cyclon at $q=0$ and $q= \pm 2 Q$. Hence we have a set of twin electromagnons at wave vectors $q=k_{0}$ and $q=k_{0}-2 Q$. For $g_{b}=g_{c}$, only one electromagnon (the zone-edge cyclon) is activated ${ }^{?}$ This excitation corresponds to a scattering process with in and out-going momenta equal to the cycloid wave vector $Q$ [Fig. 3(a)]. However, when $g_{b} \neq g_{c}$, the twin electromagnon (a cyclon at $q=k_{0}-2 Q$ ) appears, corresponding to two outgoing momenta adding up to $2 Q$ [Fig. 3(b)].

\section{DIELECTRIC FUNCTION AND COMPARISON TO EXPERIMENTS}

In order to relate to optical experiments, we calculate the dielectric function $\varepsilon(\omega)=\varepsilon_{\infty}+4 \pi P_{0}^{a}(\omega) / E^{a}$ and 


\begin{tabular}{cccccc}
\hline \hline & $\mathrm{Gd}_{0.5} \mathrm{~Tb}_{0.5}$ & $\mathrm{Gd}_{0.1} \mathrm{~Tb}_{0.9}$ & $\mathrm{~Tb}$ & $\mathrm{~Tb}_{0.41} \mathrm{Dy}_{0.59}$ & $\mathrm{Dy}$ \\
\hline$\left(\frac{\Omega_{A}}{\Omega_{B}}\right)_{\operatorname{Exp}}$ & 0.30 & 0.39 & 0.40 & 0.36 & 0.37 \\
$\left(\frac{\Omega_{c, k_{0}-2 Q}}{\Omega_{c, k_{0}}}\right)_{\mathrm{Th}}$ & 0.55 & 0.53 & 0.52 & 0.52 & 0.54 \\
$\left(\frac{\mathcal{S}_{A}}{\mathcal{S}_{B}}\right)_{\operatorname{Exp}}$ & 0.08 & 0.11 & 0.13 & 0.41 & 0.51 \\
{$\left[\frac{\left(g_{b}+g_{c}\right)^{2}}{\left(g_{b}-g_{c}\right)^{2}} \frac{\mathcal{S}_{c, k_{0}-2 Q}}{\mathcal{S}_{c, k_{0}}}\right]_{\mathrm{Th}}$} & 0.14 & 0.16 & 0.16 & 0.16 & 0.18 \\
\hline \hline
\end{tabular}

TABLE I: This table compares our theoretical calculations to experiments in $R \mathrm{MnO}_{3}$. A and $\mathrm{B}$ are, respectively, the lowerand higher-energy electromagnon modes detected in [8].

study its resonances. When the resonance frequencies are not too close to each other $\varepsilon$ is a sum of Lorentzians,

$$
\begin{aligned}
\varepsilon(\omega)= & \frac{\mathcal{S}_{k_{0}}}{\Omega_{\mathrm{C}, \mathrm{k}_{0}}^{2}-\Delta_{k_{0}}^{2}-\omega^{2}}+\frac{\mathcal{S}_{k_{0}-2 Q}}{\Omega_{\mathrm{C}, \mathrm{k}_{0}-2 \mathrm{Q}}^{2}-\Delta_{k_{0}-2 Q}^{2}-\omega^{2}} \\
& +\frac{\mathcal{S}_{\mathrm{ph}}}{\omega_{0}^{2}+\Delta_{\mathrm{ph}}^{2}-\omega^{2}}+\varepsilon_{\infty} .
\end{aligned}
$$

The electromagnon frequencies are seen to be downshifted from the bare magnon frequency $\Omega_{\mathrm{C}, \mathrm{q}}$ to $\sqrt{\Omega_{\mathrm{C}, \mathrm{q}}^{2}-\Delta_{q}^{2}}$, while the phonon frequency $\omega_{0}$ gets upshifted to $\sqrt{\omega_{0}^{2}+\Delta_{\mathrm{ph}}^{2}}$. The spectral weights $\mathcal{S}_{q}$ share a simple relationship with the frequency shifts: For elec-

An important experimental result is that the spectral weights are nearly the same for the $a b$ and $b c$ cycloids $, 7,17$ It is easy to see that this result follows from our model when $\left|g_{b}\right| \gg\left|g_{a}\right|,\left|g_{c}\right|$. Note that in this limit the magnetoelectric coupling Eq. (4) is invariant under the flip of the cycloid plane, in spite of the fact that it lacks rotational symmetry. Table I compares our theoretical calculations to the measured values $\Omega_{A} / \Omega_{B}$ and $\mathcal{S}_{A} / \mathcal{S}_{B}$, where $\mathrm{A}$ and $\mathrm{B}$ label the lower- and higherenergy electromagnons observed in RMO $\stackrel{8}{-}$ We used the same model parameters as the ones in Fig. 4(b) of Ref. [8] (filled symbols). The variations in the ratio $\mathcal{S}_{A} / \mathcal{S}_{B}$ indicate differences in the couplings $g_{c}, g_{b}$ for different ions $R$. For Tb our theory matches the observed values when $g_{c} / g_{b}=0.05 ;$ for Dy we get $g_{c} / g_{b}=-0.25$.

\section{DISCUSSION AND CONCLUSIONS}

We now consider other possibilities for the activation of the low-frequency electromagnon. Interactions containing crossed terms such as $x_{n}^{a} \hat{S}_{i}^{b} \hat{S}_{j}^{c}$ are also allowed by the Pbnm symmetry. For a $b c$ cycloid, this interaction gives rise to an electromagnon at $q=k_{0}-2 Q$. However, when the cycloid is flipped to the $a b$ plane, this interaction leads instead to an electromagnon at $q=k_{0}-Q$, with no electromagnon at $q=k_{0}-2 Q$. This result implies a large tromagnons, $\mathcal{S}_{q}=\left[\varepsilon(0)-\varepsilon_{\infty}\right] \Delta_{q}^{2}$, while for the phonon $\mathcal{S}_{\mathrm{ph}}=\left[\varepsilon(0)-\varepsilon_{\infty}\right]\left(\omega_{0}^{2}-\Delta_{\mathrm{ph}}^{2}\right)$. The frequency shifts are related by $\Delta_{\mathrm{ph}}^{2}=\Delta_{k_{0}}^{2}+\Delta_{k_{0}-2 Q}^{2}$, ensuring the satisfaction of the oscillator strength sum rule, $\mathcal{S}_{k_{0}}+\mathcal{S}_{k_{0}-2 Q}+\mathcal{S}_{\mathrm{ph}}=$ $\omega_{0}^{2} \chi_{0}$. The electromagnon spectral weights are given by

$$
\begin{gathered}
\mathcal{S}_{k_{0}}=\frac{4 \pi S \chi_{0}^{2} v_{0} \omega_{0}^{4}\left(g_{b}+g_{c}\right)^{2}}{\left(\omega_{0}^{2}-\Omega_{c, k_{0}}^{2}\right)^{2}} \tan ^{2} \frac{Q b}{2}\left(\frac{\Omega_{\mathrm{C}, \mathrm{k}_{0}}^{2}}{J_{2 b}}\right), \\
\mathcal{S}_{k_{0}-2 Q}=\frac{2 \pi S \chi_{0}^{2} v_{0} \omega_{0}^{4}\left(g_{b}-g_{c}\right)^{2} \tan ^{2} \frac{Q b}{2}}{\left(\omega_{0}^{2}-\Omega_{c, k_{0}-2 Q}^{2}\right)^{2}\left[\cos ^{4} \frac{Q b}{2}+\sin ^{4} \frac{Q b}{2}\right]}\left(\frac{\Omega_{\mathrm{C}, \mathrm{k}_{0}-2 \mathrm{Q}}^{2}}{J_{2 b}}\right) .
\end{gathered}
$$

shift in electromagnon frequency (more than $50 \%$ as seen in Fig. 2), that is in contradiction to experiments $7,8,9,17$ Therefore the interaction $x_{n}^{a} \hat{S}_{i}^{b} \hat{S}_{j}^{c}$ can not explain the origin of the low-frequency electromagnon. Similarly, interactions of the form $x_{n}^{a} \hat{S}_{i}^{a} \hat{S}_{j}^{c}$ only give rise to electromagnons at $k_{0}-Q$; this leads to ratios of electromagnon frequencies $\Omega_{A} / \Omega_{B}$ that are twice as large as obtained experimentally (Table I) $\stackrel{8}{*}$ Hence $x_{n}^{a} \hat{S}_{i}^{a} \hat{S}_{j}^{c}$ is also ruled out.

Aguilar et $a l^{\frac{7}{7}}$ suggested that an elliptical spiral structure such as the one in $\mathrm{BiFeO}_{3}$ (Refs. 6 and 19) may explain the low-frequency electromagnon. This possibility is also ruled out because the degree of ellipticity observed in neutron-scattering experiments 11 is too low to explain the large spectral weight of the low frequency electromagnon. Furthermore, Ref. 7 suggested that purely magnetic interactions such as $\hat{S}_{i}^{a} \hat{S}_{j}^{c}$ would mix extra-cyclon magnons at $q=Q$ to the zone-edge electromagnon, providing an alternative explanation for the low frequency resonance. However, the extra-cyclon magnon energy at $q=Q$ is approximately constant for different $R$ 's, in contradiction to the trend observed in experiments $\left[\Omega_{A}\right.$ tends to decrease with increasing ionic radius; $\Omega_{A}$ for $\mathrm{Gd}_{0.5} \mathrm{~Tb}_{0.5}$ is $40 \%$ higher than for Dy (Ref. 8) ]. Interestingly, our theory gives a natural explanation for this trend since the value of the cyclon energy at $q=k_{0}-2 Q$ also decreases appreciably as the ionic radius increases. 
We now discuss the implications of our model for the understanding of multiferroic order. One remarkable consequence of the presence of the $q=k_{0}-2 Q$ electromagnon is that its spectral weight can be directly related to the presence of the IOP in the ground state. Using Eqs. (6) and (12b) we derive an important relation between the amplitude of the IOP and the twin electromagnon spectral weight,

$$
P_{\mathrm{IOP}}^{2}=\frac{8 S^{3} J_{2 b}}{\pi v_{0}} \cos ^{2} \frac{Q b}{2}\left[\cos ^{4} \frac{Q b}{2}+\sin ^{4} \frac{Q b}{2}\right] \frac{\mathcal{S}_{k_{0}-2 Q}}{\Omega_{k_{0}-2 Q}^{2}} .
$$

Hence optical experiments are a direct probe of the IOP amplitude. For $\mathrm{TbMnO}_{3}$, the measured twin electromagnon frequency is $\Omega_{\mathrm{C}, \mathrm{k}_{0}-2 \mathrm{Q}}=25 \mathrm{~cm}^{-1}$, with spectral weight $\mathcal{S}_{k_{0}-2 Q}=1.7 \times 10^{3} \mathrm{~cm}^{-2} \underline{\underline{8}}$ Using Eq. (13) we obtain $P_{\mathrm{IOP}}=4 \mu \mathrm{C} / \mathrm{cm}^{2}$, a value that is 50 times larger than the uniform polarization $P_{0}=8 \times 10^{-2} \mu \mathrm{C} / \mathrm{cm}^{2}$ present in $\mathrm{TbMnO}_{3}$.

In conclusion, we introduced the concept of the twin electromagnon in order to explain optical experiments in the $R \mathrm{MnO}_{3}$ family of multiferroics. Our symmetry analysis shows that an incommensurate oscillatory polarization coexists with the well-known cycloid phase in these materials. Remarkably, there exists a direct relation between the twin electromagnon spectral weight and the amplitude of this oscillatory polarization. Hence we showed that $\mathrm{TbMnO}_{3}$ has an oscillatory polarization with amplitude 50 times larger than its uniform polarization. This surprising conclusion underlines the importance of electromagnons in the characterization of multiferroic order.

\section{ACKNOWLEDGMENTS}

We thank N. Kida and A. Pimenov for useful discussions. This research was supported by NSERC discovery and the UVic Faculty of Sciences.
* markku.stenberg@iki.fi

$\dagger$ rdesousa@uvic.ca

1 T. Kimura, T. Goto, H. Shintani, K. Ishizaka, T. Arima, and Y. Tokura, Nature 426, 55 (2003).

2 G.A. Smolenskii and I.E. Chupis, Sov. Phys. Usp. 25, 475 (1982).

3 V.G. Bar'yakhtar and I.E. Chupis, Sov. Phys. Solid State 10, 2818 (1969); V.G. Bar'yakhtar and I.E. Chupis, Sov. Phys. Solid State 11, 2628 (1970).

4 A. Pimenov, A. A. Mukhin, V. Y. Ivanov, V. D. Travkin, A. M. Balbashov, and A. Loidl, Nature Phys. 2, 97 (2006).

5 A. B. Sushkov, R. Valdés Aguilar, S. Park, S-W. Cheong, and H. D. Drew, Phys. Rev. Lett. 98, 027202 (2007).

6 M. Cazayous, Y. Gallais, A. Sacuto, R. de Sousa, D. Lebeugle, and D. Colson, Phys. Rev. Lett. 101, 037601 (2008).

7 R. Valdés Aguilar, M. Mostovoy, A. B. Sushkov, C. L. Zhang, Y. J. Choi, S-W. Cheong, and H. D. Drew, Phys. Rev. Lett. 102, 047203 (2009).

8 J.S. Lee, N. Kida, S. Miyahara, Y. Takahashi, Y. Yamasaki, R. Shimano, N. Furukawa, and Y. Tokura, Phys. Rev. B 79, 180403(R) (2009).

9 N. Kida, Y. Ikebe, Y. Takahashi, J. P. He, Y. Kaneko, Y. Yamasaki, R. Shimano, T. Arima, N. Nagaosa, and Y. Tokura, Phys. Rev. B 78, 104414 (2008).

10 D. Senff et al., Phys. Rev. Lett. 98, 137206 (2007).

11 M. Kenzelmann, A. B. Harris, S. Jonas, C. Broholm, J. Schefer, S. B. Kim, C. L. Zhang, S.-W. Cheong, O. P. Vajk, and J. W. Lynn, Phys. Rev. Lett. 95, 087206 (2005).
12 M. Mostovoy, Phys. Rev. Lett. 96, 067601 (2006).

13 H. Katsura, N. Nagaosa, and A.V. Balatsky, Phys. Rev. Lett. 95, 057205 (2005).

14 I. A. Sergienko and E. Dagotto, Phys. Rev. B 73, 094434 (2006).

15 A. Malashevich and D. Vanderbilt, Phys. Rev. Lett. 101, 037210 (2008).

${ }^{16}$ H. Katsura, A. V. Balatsky, and N. Nagaosa, Phys. Rev. Lett. 98, 027203 (2007).

17 A. Pimenov, A. Shuvaev, A. Loidl, F. Schrettle, A. A. Mukhin, V. D. Travkin, V. Yu. Ivanov, and A. M. Balbashov, Phys. Rev. Lett. 102, 107203 (2009).

18 A third, lower frequency electromagnon with quite small spectral weight was reported in 17 .

19 R. de Sousa and J. E. Moore, Phys. Rev. B 77, 012406 (2008).

20 E.M. Lifshitz and L.P. Pitaevskii, Statistical Physics Part 2, vol. 9 of Landau and Lifshitz Course of Theoretical Physics (Butterworth-Heinemann, Oxford, U.K., 1980).

21 M. D. Glinchuk, E. A. Eliseev, A. N. Morozovska, and R. Blinc, Phys. Rev. B 77, 024106 (2008).

22 R. Kajimoto, H. Mochizuki, H. Yoshizawa, H. Shintani, T. Kimura, and Y. Tokura, J. Phys. Soc. Jpn. 74, 2430 (2005).

23 There exists a linear relation connecting the fields $\psi_{i n}$ to the fields $\phi_{i n}$; therefore the $\psi_{i n}$ are completely specified once the $\phi_{\text {in }}$ are set. 